\title{
BIODIVERSIDAD DE MACROINVERTEBRADOS BENTÓNICOS EN EL SISTEMA DE CULTIVO DE ARROZ EN EL SECTOR MUÑUELA MARGEN DERECHO EN PIURA, PERÚ
}

\section{BIODIVERSITY OF BENTHIC MACROINVERTEBRATES IN A RICE FIELD SYSTEM AT THE MUÑUELA MARGEN DERECHO SECTOR, PIURA, PERU}

\author{
Florencia Andrea Trama ${ }^{1}$ y Jesús Abel Mejía Marcacuzco²
}

\begin{abstract}
Resumen
El presente estudio tuvo por objetivo describir la estructura y composición de las comunidades de macroinvertebrados presentes en partes del sistema de riego y en parcelas de cultivo de arroz del sector Muñuela Margen Derecho en el Bajo Piura, Perú. Se evaluó la colonización de macroinvertebrados tomando en sustratos artificiales muestras en tres puntos principales del sistema de canales: P1 (entrada de agua de riego), P2 (drenaje principal) y P3 (canal que desemboca en los Manglares de San Pedro de Vice) y en tres parcelas de arroz individuales del sector de riego seleccionado. Los resultados indican que las comunidades de macroinvertebrados variaron entre los tres puntos de muestreo principales y entre las tres parcelas de arroz: En el punto P1 se encontró una riqueza media de familias de 11, una riqueza media de especies de 16, la diversidad con el índice de Shannon-Wiener de 2.24 y una abundancia media de macroinvertebrados de 450, siendo estos valores mayores que en P2 y P3 (siempre con valores de $\mathrm{P} \leq 0,01)$. Las parcelas de arroz evaluadas individualmente presentaron diferencias entre sí, pero siempre mostraron valores de riqueza, diversidad y abundancia menores que en los puntos principales de muestreo. Algunos de los parámetros fisicoquímicos (conductividad eléctrica, salinidad y oxígeno disuelto) y nutrientes evaluados (fósforo y fuentes de nitrógeno inorgánico total) explican las respuestas observadas en la variación de la estructura y composición de las comunidades de macroinvertebrados entre los diferentes puntos de muestreo evaluados. Los resultados del presente estudio dan a conocer una primera aproximación a las comunidades de macroinvertebrados en estos agroecosistemas y en el sistema de riego, que en el futuro podrían ser utilizados como indicadores de la calidad de agua para mejorar las prácticas de manejo del cultivo.

Palabras clave: agroecosistemas, macroinvertebrados, arroz, riego, Piura.
\end{abstract}

\begin{abstract}
The objective of this study was to describe the structure and composition of aquatic macroinvertebrate communities in the irrigation system and rice fields of the Muñuela Margen Derecho sector in the low Piura, Peru. The colonization of macroinvertebrates in artificial substrates was evaluated in three main sampling points (water entrance) P1, P2 (main drain) and P3 (outlet channel at the Manglares de San Pedro de Vice). We also evaluated three rice fields within the selected irrigation system as well as in three main sites and in three rice plots: site P1 presented a mean family richness of 11 , a mean species richness of 16 , a diversity with the index of Shannon-Wiener of 2.24 and a mean abundance of macroinvertebrates of 450 greater than P2 and P3 (always with P values $<0.01$ ); individual rice plots presented differences between them, but always had values of richness, diversity and abundance below the values at the main sampling sites. Some physicochemical parameters (conductivity, salinity and dissolved oxygen) and evaluated nutrients (phosphorus and total inorganic nitrogen sources) explained the observed responses in the variation of structure and composition of the communities of aquatic macroinvertebrates between different sampling sites assessed. The results of this study show a first approximation to the macroinvertebrate communities in these agro-ecosystems and at the irrigation system, which could be used as indicators of water quality to improve crop management practices in the future.
\end{abstract}

Key words: agroecosystems, macroinvertebrates, rice, irrigation, Piura. 


\section{Introducción.}

Los cultivos de arroz son agroecosistemas, tropicales o subtropicales, altamente ricos en fauna y flora (Curcó et al., 2001; FAO, 2004; Blázquez, 2005) debido a que las características físicas y químicas del agua cambian constantemente durante el cultivo y son muy similares a los humedales naturales (Rizo-Patrón, 2003; Blanco et al., 2006; Rizo-Patrón \& Trama, 2008; Trama et al., 2008; Acosta et al., 2010; RizoPatrón et al., 2013). En estos agroecosistemas los invertebrados son los organismos más abundantes y con mayor diversidad biológica, los cuales han sido estudiados en diversos cultivos por su importancia en la cadena trófica y como indicadores de contaminación (Curcó et al., 2001; Roldán, 2003; Dominguez \& Fernandez, 2009). La fuente de irrigación afecta directamente a la ecología y diversidad de estos ecosistemas y la fuente del agua determina las características fisicoquímicas y la composición de la biota (Bambaradeniya, 2000; Bambaradeniya \& Amarasinghe, 2003; Blanco, 2011).

En el Perú se han realizado varios trabajos sobre invertebrados acuáticos (Iannacone \& Alvarino, 2002; Martínez, 2002; Iannacone et al., 2003; Paredes et al., 2007; Acosta et al., 2009; Dominguez \& Fernandez, 2009; Prat et al., 2009; Huamantico \& Ortiz, 2010). Sin embargo, no se han encontrado publicaciones con referencia a las comunidades de macroinvertebrados bentónicos en los cultivos de arroz bajo riego o los canales de riego y drenaje asociados a estos agroecosistemas. Por lo tanto, el objetivo del presente estudio fue realizar una caracterización inicial de las comunidades de macroinvertebrados acuáticos en parcelas de arroz e infraestructura de riego asociada a la comisión de riego Muñuela Margen Derecha en Piura, norte de Perú.

Objetivo.

Caracterizar a las comunidades de macroinvertebrados bentónicos presentes en el sistema de riego y los arrozales presentes en la comisión de regantes Muñuela Margen Derecha, Sechura, Piura. Área de estudio.

La investigación se llevó a cabo en el distrito de Vice que se encuentra ubicado en la Provincia de Sechura, Departamento de Piura al noroeste del Perú (5²5'13.03"S 8046'26.73"O). El área evaluada pertenece a la región de Desierto Costero y de acuerdo con el Sistema de zonas de vida de Holdridge y el Mapa Ecológico del Perú, pertenece a la provincia de Zonas Áridas (INRENA, 1994). Debido a su ubicación geográfica, el área se caracteriza por un clima extremadamente seco con precipitaciones muy escasas (menos de $50 \mathrm{~mm} / \mathrm{año}$ ). La temperatura media anual es de $26^{\circ} \mathrm{C}$ (Amorós \& Ota, 2002).

\section{Materiales y métodos.}

Los muestreos se realizaron en la cuenca baja del río Piura, en el sector de Sechura-Vice, específicamente en la infraestructura bajo control de la Comisión de Regantes Muñuela Margen Derecha (Figura 1). El sistema de irrigación incluye canales de riego, principales y secundarios provenientes del reservorio Poechos, y canales de drenaje que toman las aguas de cada parcela y las llevan hasta su desembocadura en la parte baja de la cuenca. El dren Sechura desemboca en el mar formando un estuario en el cual se ha desarrollado el ecosistema natural Manglares de San Pedro de Vice, el cual recibe todas las aguas de drenaje de los cultivos de la zona. Este humedal es clave para la vida silvestre por proporcionar gran cantidad de hábitats (TabiloValdivieso et al., 1999).

El trabajo de campo se llevó a cabo durante los meses de agosto a diciembre de 2010 durante los meses que dura la campaña chica de arroz. Los muestreos se realizaron en tres puntos principales, Canal de riego (P1), Canal de drenaje principal (P2) y Canal Sechura (P3). Asimismo, se realizaron muestreos en 3 parcelas individuales de arroz (VAC, MVI y JTT) incluyendo entradas y salidas del agua de riego (Tabla 1, Figura 1). Se utilizó el método de sustratos artificiales (Castillo, 2000) que consiste en colocar bloques de concreto $(20 \times 5 \times 10 \mathrm{~cm})$ dentro de una bolsa de malla plástica y colocarlos aproximadamente a $50 \mathrm{~cm}$ de profundidad durante un mes para permitir que los macroinvertebrados los colonicen (Trama et al., 2009). En cada estación de muestreo se ubicaron tres bloques (repeticiones), y se realizaron 4 muestreos (uno cada mes). Se colocaron un total de 36 sustratos artificiales en los puntos principales y en las tres parcelas de arroz evaluadas se colocaron tres sustratos en las entradas y tres en las salidas de agua, haciendo un total de 72 sustratos durante todo el muestreo (Tabla 1, Figura 1). Para la recolecta de los invertebrados se lavaron tanto los bloques como las bolsas y después se pasó la muestra por un tamiz de $500 \mu \mathrm{m}$ (Castillo, 2000). Las muestras fueron conservadas en alcohol al 70\% para luego llevarlas al laboratorio y ser analizadas. En el laboratorio, cada muestra se lavó con agua y se tamizó nuevamente para eliminar todas las partículas de sedimento que pudieran haber quedado. Los organismos se analizaron utilizando una Lupa Binocular Estereoscópica 3X (ARCANO) y un microscopio 40X (Olympus) separando los individuos según su parecido morfológico (morfo especies) y luego se clasificaron por medio de las claves generadas por especialistas en un compendio general (Dominguez \& Fernandez, 2009). Se define "taxa" como el número total de familias, géneros o especies identificadas para un muestreo determinado. Cuando se identifica una especie, la misma vale como un taxa, pero no se incluye al género o la familia en el conteo para no sobreestimar las variables de riqueza. Además, de los muestreos biológicos, se tomaron muestras de agua en botellas de plástico y fueron refrigeradas para 
evaluar nutrientes en laboratorio (Nitratos, Nitritos, Amonio, Potasio y Fosfatos). En el sitio se midieron datos fisicoquímicos por triplicado considerando: $\mathrm{pH}$ mediante un medidor portátil (Adwa), el oxígeno disuelto, salinidad, temperatura y conductividad usando un equipo multiparamétrico (YSI-85), profundidad con una regla y transparencia mediante un disco Secchi dentro de un tubo de $1 \mathrm{~m}$ de alto, para luego realizar la conversión a NTU para estimar la turbidez.

Análisis de los datos

1. Macroinvertebrados: Luego de identificar a los macroinvertebrados se contaron los individuos y especies para cada muestra y en cada punto de muestreo/fecha. Con esos datos se realizaron tres tipos de análisis tanto en los puntos principales como en las parcelas de arroz individuales:

a. Análisis de valores totales: En cada punto de muestreo se estimó un valor total de riqueza de familias (sumando el número de familias presentes en todo el estudio sin repetir), un valor total de riqueza de taxa (sumando el número de taxa presentes en todo el estudio sin repetir), y un valor total de abundancia de macroinvertebrados (sumando el número de invertebrados en todo el estudio).

b. Análisis de valores promedio: Para cada punto de muestreo se estimó un valor medio de las 3 repeticiones (bloques). Se estimó la riqueza media de familias, la riqueza media de taxa y la abundancia media de macroinvertebrados. Se realizaron análisis de varianza paramétricos usando el programa Statgraphics Centurión XV (Statpoint Technologies, 2007) para comparar las variables estimadas entre los distintos puntos de muestreo.

c. Índices de estructura: Se calculó el índice de diversidad de Shannon-Wiener ( $\left.\mathrm{H}^{\prime}\right)$ y el índice cuantitativo de similaridad de Jaccard (Moreno, 2001). Por otro lado, se determinó la estructura y composición de la comunidad de macroinvertebrados para cada orden y en cada punto de muestreo.

Nutrientes y Fisicoquímicos: Los resultados de los nutrientes fueron analizados para cada uno de los puntos principales y meses evaluados y así determinar las tendencias. Para los parámetros fisicoquímicos se estimaron los valores medios en cada punto de muestreo y fecha, los cuales al igual que en el caso de nutrientes, fueron organizados en tablas y analizados para determinar las tendencias.

\section{Resultados.}

Durante el estudio se registraron en total 46 familias de invertebrados con 81 taxa (género o especie) y un total de 7916 individuos tanto para los puntos principales como para las parcelas individuales (Tabla 2). En el sistema de riego se registraron en total 32 taxa de macroinvertebrados para el punto $\mathrm{P} 1,31$ taxa para el P2 y 15 taxa para el P3. En las parcelas individuales se contabilizaron 44 taxa para la parcela
VAC, 22 para la parcela MVI y 18 taxa para la parcela JTT. Macroinvertebrados en el Sistema de Riego

La riqueza media de familias de macroinvertebrados fue siempre mayor en el punto P1 que en el P2 y que en el P3 para todo el estudio en general $(F=18,90 ;$ gl=2,35; $P=0,0001$ ) (Figura 2a) y sin mostrar diferencias significativas durante los 4 meses evaluados ( $\mathrm{F}=0,72 ; \mathrm{gl}=3,35 ; \mathrm{P}=0,5519)$. Asimismo, la riqueza media de taxa de macroinvertebrados fue mayor en el punto P1 que en P2 y P3 ( $F=22,67 ; \mathrm{gl}=2,35 ; \mathrm{P}=0,0001)$ sin mostrar diferencias en los muestreos mensuales $(\mathrm{F}=0,67$; $\mathrm{gl}=3,35 ; \mathrm{P}=0,5807$ ) (Figura 2b). La abundancia media de macroinvertebrados fue mayor en el punto P1 que en el P2 y en el P3 para todo el estudio, mostrando diferencias significativas entre P1 y P2 y P1 y P3 $(\mathrm{F}=5,52 ; \mathrm{gl}=2,35 ; \quad \mathrm{P}=0,01)$ pero sin mostrar diferencias significativas entre los meses evaluados $(\mathrm{F}=1,71$; $\mathrm{gl}=3,35 ; \mathrm{P}=0,1907$ ) (Figura 2c). Los valores totales mostraron una tendencia similar a la observada en los análisis de las medias. En los puntos principales del sistema de riego se registraron mayores valores totales de riqueza de familias y de taxa en los puntos P1 y P2. En el caso de la abundancia total se observó que el P1 y el P3 presentaron mayor cantidad de individuos que P2 (Tabla 2). El índice de diversidad de Shannon-Wiener ( $\left.H^{\prime}\right)$ fue mayor para P1 $(2,24)$ y P2 $(2,47)$ que para P3 $(0,86)$. El índice de similaridad de Jaccard (Ij) mostró que los tres puntos compartieron menos del $50 \%$ de las especies de macroinvertebrados entre sí: P1 y P2: 38\%, P1 y P3: 28\% y P2 y P3: $42 \%$.

Los órdenes con mayor diversidad en términos de riqueza de especies de macroinvertebrados fueron Trichoptera (23\%), Diptera (17\%) y Coleoptera (12\%) para P1, Coleoptera (19\%), Diptera (13\%) y Hemiptera, Odonata y Basomatophora con 10\% cada uno en P2, y Basomatophora, Díptera y Odonata con 19\% para P3. Los grupos más abundantes, en cambio, fueron Trichoptera (42\%) y Diptera (25\%) para P1, Basomatophora (41\%) y Diptera (14\%) en P2, y, Diptera (57\%) y Basomatophora (14\%) para P3 (Tabla 3).

Macroinvertebrados en las parcelas individuales de arroz

Las parcelas de arroz individuales, fueron similares respecto al manejo del cultivo (Tabla 4). La riqueza de familias y de taxa fueron diferentes no así en el caso de la abundancia. En términos generales y para todo el estudio, la riqueza media de familias de macroinvertebrados fue mayor en la parcela VAC que en la JTT y que en la MVI ( $\mathrm{F}=14,92 ; \mathrm{gl}=2,54$; $\mathrm{P}$ $=0,0001)$ y varió entre meses $(F=10,14 ; \mathrm{gl}=3,54 ; \mathrm{P}$ =0,0001), no así entre los puntos de entrada y salida (Figura 2d). De igual forma, la riqueza media de taxa de macroinvertebrados fue mayor en la parcela VAC, que en la MVI y que en la JTT ( $F=22,77$; gl=2,54; P 
$=0,0001)$ y también fue diferente entre los meses evaluados $(\mathrm{F}=7,81 ; \mathrm{gl}=3,54 ; \mathrm{P}=0,0002)$, pero no entre los puntos de entrada y salida (Figura 2e). Al igual que en los puntos principales, los valores totales estimados al sumar todos los taxa de macroinvertebrados en cada parcela mostraron una tendencia similar a la observada en el análisis medio (Tabla 2). El índice de diversidad de Shannon-Wiener $\left(\mathrm{H}^{\prime}\right)$ mostró valores similares entre las tres parcelas, ligeramente mayor en MVI (1.49) que en VAC (1.32) y que en JTT (1.16). Sin embargo, el índice de similaridad de Jaccard (Ij) mostró que los tres puntos compartieron pocas especies entre sí. Las parcelas VAC y MVI compartieron el $40 \%$ de las especies de macroinvertebrados, mientras que VAC y JTT solo 25\% y JTT y MVI el $22 \%$.

Los órdenes con mayor diversidad en términos de riqueza de taxa de fueron Diptera (29\%) y Coleoptera (21\%) para la parcela VAC, Diptera (29\%), Basomatophora (26\%) y Coleoptera (13\%) para JTT y Diptera (29\%), Basomatophora (14\%) y Coleoptera (21\%) para MVI. Asimismo, los grupos más abundantes fueron Diptera (59\%) y Basomatophora (26\%) para VAC y Basomatophora (62\%) Ostradoda y Diptera (18\%) cada uno en JTT, y Diptera (28\%) y Basomatophora (45\%) en MVI (Tabla 3). Por último, las tres parcelas de forma individual no mostraron diferencias significativas para las variables de riqueza de familias, riqueza de taxa o abundancia de macroinvertebrados entre las entradas y las salidas de las tres parcelas evaluadas.

Factores fisicoquímicos y concentración de nutrientes en los puntos principales

Los valores de los parámetros fisicoquímicos variaron en algunos casos entre los diferentes puntos principales y los meses evaluados (Tabla 5). Específicamente, la temperatura varió entre 20.5 y 28 unidades en todos los puntos principales sin mostrar diferencias significativas ( $\mathrm{F}=0,17 ; \mathrm{gl}=2,14 ; \mathrm{P}=0,842$ ) al igual que la turbidez y la temperatura $(\mathrm{F}=1,13$; $\mathrm{gl}=2,14 ; \mathrm{P}=0,35)$ y $(\mathrm{F}=2,39 ; \mathrm{gl}=2,14 ; \mathrm{P}=0,1335)$. Por otro lado, el $\mathrm{pH}$ fue ligeramente mayor para el punto P1 que para P2 y P3 ( $F=5,62 ; g l=2,14 ; P=0,019)$. La conductividad eléctrica se mantuvo por debajo de 1 000,0 $\mu \mathrm{S}$ en el punto P1 y entre 4 000,0 a 7 000,0 unidades para los puntos P2 y P3, observándose diferencias significativas entre los puntos P1 y P2 y P1 y P3 ( $\mathrm{F}=11,74 ; \mathrm{gl}=2,14 ; \mathrm{P}=0,0019)$. El oxígeno disuelto mostró diferencias significativas entre P1 y P2 (F=3,99; $g l=2,14 ; P=0,047)$. Por último, la salinidad se mantuvo baja en P1 (menor a 0.5 ppm) y alrededor de $3 \mathrm{ppm}$ en los puntos P2 y P3 observándose diferencias significativas entre P1 y P2 y P1 y P3 ( $F=11,64 ;$ gl=2,14; $\mathrm{P}=0,0019)$. No se observaron diferencias significativas para ninguna de las variables fisicoquímicas durante los cuatro meses evaluados.
Para el caso de los nutrientes, en P1, los fosfatos fueron altos en el primer mes de estudio y luego disminuyeron en los meses evaluados en el orden de 10 a 15 veces. Sin embargo, en P2 y P3 tendieron a un ligero aumento (Tabla 6). El potasio aumentó ligeramente en el punto P1 y P3 durante todo el estudio mientras que en P2 disminuyó ligeramente. Los nitritos disminuyeron fuertemente a lo largo del estudio en el punto P2, ligeramente en el punto P1 y aumentaron también ligeramente en P3. El amonio aumentó en el punto P3 y disminuyó en los puntos P1 y P2 durante el estudio. Los nitratos aumentaron en P2 a lo largo de los meses en el orden de 4 a 10 veces. En P3 tendieron a disminuir ligeramente y en P1 los valores se mantuvieron estables. Por último, las formas inorgánicas del N sumadas (nitritos, nitratos y amonio) se combinaron para evaluar la tendencia general del elemento. Este valor aumentó fuertemente en el P2 a lo largo del estudio.

Factores fisicoquímicos en las parcelas de arroz

En la parcela VAC y JTT ninguno de los parámetros fisicoquímicos medidos mostró diferencias significativas entre la entrada y la salida de las parcelas (Tabla 5). En la parcela MVI solo se observaron diferencias significativas entre la entrada y la salida de la parcela para la conductividad eléctrica y salinidad. Por otro lado, se observaron diferencias significativas para el parámetro de conductividad entre JTT y MVI, y para profundidad y salinidad entre JTT y VAC y JTT y MVI. No se observaron diferencias significativas para ninguna de las variables fisicoquímicas durante los cuatro meses evaluados.

\section{Discusión.}

El presente estudio presenta la primera descripción de la comunidad de macroinvertebrados de los arrozales del bajo Piura y la primera lista taxonómica en el sistema de irrigación y de tres parcelas de arroz del sector de Muñuela Margen Derecha en el Bajo Piura.

Las comunidades de macroinvertebrados variaron dentro del sistema de riego artificial

La riqueza de familias, riqueza de taxa y abundancia de macroinvertebrados variaron en los tres puntos de muestreo principales. En términos generales la riqueza fue mayor tanto en promedio como en valores totales en el punto de entrada de riego (P1), que en los puntos $\mathrm{P} 2$ y que en $\mathrm{P} 3$ respectivamente. A pesar de que la riqueza de familias y taxa de macroinvertebrados fue siempre mayor en el punto P1, el índice de diversidad de Shannon-Wiener fue mayor en P2. Esto puede deberse a que las abundancias de los diferentes taxa para el punto P2 han sido más uniformes que en el P1, dando como resultado un índice mayor (Moreno, 2001; Chao \& Shen, 2009). El índice de similaridad de Jaccard mostró que las especies compartidas fueron pocas, menos del $50 \%$. Estos resultados de cambio en la riqueza y diversidad, 
pueden responder además a la concentración diferencial de nutrientes. El punto P1 (presumiblemente sin agroquímicos) presentó una menor concentración de nutrientes para casi todos los meses excepto en el mes de agosto, donde el fosfato fue mas alto y en octubre donde las fuentes inorgánicas de nitrógeno en total también fueron mas altas. El punto P2, tuvo una mayor concentración de nutrientes y cambios en la conductividad, $\mathrm{pH}$, salinidad y oxígeno disuelto (Tabla 5), y por último el $\mathrm{P} 3$, aguas del canal que se dirige al ecosistema de Manglar con una probable dilución resultado de la convergencia con las aguas del río Piura (Figura 1). Es posible que los agroquímicos detectados en P1 provengan del embalse "Los Ejidos", que colecta agua desde la cuenca, o podría ser proveniente del lavado de las mochilas de fumigación en los canales de riego. Esto implica que el agua ya llega al sistema de riego estudiado con cierta concentración de agroquímicos. El aumento de la concentración de nutrientes en agua luego de cultivos agrícolas frecuentemente está asociado a una disminución de la calidad de agua, lo que a la vez condiciona el establecimiento de diferentes ordenes de macroinvertebrados (Harding et al., 1999). Adicionalmente, parte de los nutrientes pueden quedar por décadas en los arrozales y ser liberados con el tiempo a los cuerpos de agua cercanos (Comin et al., 2001).

Asimismo, la estructura y composición de la comunidad de macroinvertebrados parecen haber sido influenciadas por el sistema de riego. Los tres puntos de muestreo compartieron menos de $50 \%$ de las especies entre ellas, y presentaron algunos taxa exclusivos (P1: Tricorythodes sp., Macrelmis sp., Limnocoris sp., Pyraustinae sp. Glossosomatidae sp., Neotrichia sp., Nectopsyche sp.1, Macronema sp., Oecetis sp., Simulium sp. Hemerodromia sp. Ostracoda sp.1 y sp. 2, y Conchostraca sp.1.; P2; Hebridae sp. 1 y sp. 2, Celina sp., Ptilodactylidae sp., Curculionidae sp., Haliplidae sp., Alluaudomyia sp., Acaridae sp.1, sp.2 y sp.3, y para P3: Baetis sp., Scatella sp., fueron los únicos taxa exclusivos. En el caso del punto P1 la presencia de taxa "raros" (hasta 5 individuos) incrementaron la riqueza de macroinvertebrados por sobre los otros dos tratamientos. Los taxa encontrados en el punto P1 suelen hallarse en aguas con baja carga orgánica, con valores de conductividad bajos y oxígeno disuelto altos. En cambio, los taxa encontrados en los puntos P2 y P3 suelen hallarse en aguas con mayor carga de nutrientes y altos valores de conductividad (Bambaradeniya, 2000; Bambaradeniya \& Amarasinghe, 2003; Azrina et al., 2006). Por otro lado, la abundancia media fue mayor en P1 (más del doble que para P2 y P3) para todo el estudio, mientras que la abundancia total (todos los individuos colectados para el estudio) fue mayor en P1 que en P3 $\mathrm{y}$ que en P2. Estos resultados indican que las condiciones de hábitat para los macroinvertebrados son mejores en el punto de riego. Sin embargo, en P3, es posible que algunos grupos de macroinvertebrados respondan positivamente a la alta concentración de nutrientes, los cuales pueden influir notablemente la productividad primaria y como resultado estas especies tengan grupos con grandes tamaños poblacionales (Bambaradeniya \& Amarasinghe, 2003).

Manejo de la parcela de arroz condiciona la estructura y composición de macroinvertebrados

Las diferencias observadas en la riqueza de familias y taxa de macroinvertebrados entre las parcelas evaluadas podrían ser explicadas por las diferencias en el tratamiento del cultivo de arroz, tanto en la aplicación de plaguicidas como de agroquímicos (nutrientes y aditivos). Esto se reflejaría en una mayor aplicación de nutrientes (urea y fosfatos) en la parcela MVI en comparación con las otras dos evaluadas, lo que coincide con una abundancia de casi el doble de macroinvertebrados en esta parcela. Más aún los macroinvertebrados abundantes en la parcela MVI (con mayor aplicación de agroquímicos) corresponden al orden Basomatophora, los cuales suelen indicar condiciones de eutroficación por exceso de nutrientes (Roldán, 1999; Roldán, 2003).

Por otro lado, las condiciones fisicoquímicas fueron diferentes entre entrada y salida para MVI en el caso de la conductividad y la salinidad. La riqueza de familias y de taxa menor en la parcela JTT podría estar asociada a una baja diversidad de micro hábitats o tal vez a la aplicación de cipermetrina justo antes del muestreo. Al igual que en los puntos principales P2 y $\mathrm{P} 3$, los ordenes más ricos y abundantes encontrados en las parcelas individuales corresponden ordenes que frecuentemente se encuentran en sitios con mayor concentración de nutrientes, lo cual incrementa la productividad primaria y favorece la abundancia de los macroinvertebrados que se alimentan de algas como los moluscos (Basomatophora) (Leitão et al., 2007). Asimismo, las condiciones fisicoquímicas en las parcelas difieren de las encontradas en el punto $\mathrm{P} 1$, con una conductividad media mayor $\mathrm{y}$ profundidad media menor. Estos factores pueden influenciar negativamente la posibilidad de colonización por parte de adultos de algunos órdenes que se no se han observado en las parcelas de arroz.

\section{Conclusiones.}

Los datos de este estudio indican que el sistema de riego-drenaje en el sector Muñuela Margen Derecha afectó negativamente a las comunidades de macroinvertebrados en el punto P2 en comparación con P1. Esto se puede evidenciar en: 1) el punto P1 tuvo siempre mayores valores de riqueza de familias y taxa, tanto total como media, 2) un índice de ShannonWeiner mayor en el punto P1 que en P2, 3) la presencia de especies sensibles de macroinvertebrados 
en P1 (Tricorythodes sp., Macrelmis sp., Limnocoris sp., Pyraustinae sp. Glossosomatidae sp., Neotrichia sp., Nectopsyche sp.1, Macronema sp., Oecetis sp.,) y 4) una abundancia mayor en general para la mayoría de los órdenes en el agua de riego en comparación con el drenaje general. Por otro lado, la comunidad de macroinvertebrados en el punto P3 estaría influenciada por la dilución de los contaminantes y un cambio en las condiciones fisicoquímicas producto de la confluencia de las aguas de drenaje de los cultivos con otras aguas provenientes del río Piura y posiblemente por efectos de las mareas provenientes del estuario del Manglar de San Pedro de Vice.

En las parcelas individuales de arroz en cambio, la riqueza y diversidad de macroinvertebrados siempre fue menor que en los puntos principales de muestreo, especialmente que P1 y P2. Los valores de diversidad fueron menores indicando que las parcelas como tal, ofrecerían hábitat adecuado solo para algunos grupos de macroinvertebrados y no para todos los que podrían establecerse en el área. En la zona frecuentemente se elimina la vegetación de los canales para facilitar el drenaje, sin embargo se recomienda mantener zonas con plantas de Typha sp. en los canales de drenaje, las cuales son muy buenas captadoras de nutrientes, especialmente N y P (Comin et al., 2001). Esto puede ayudar a remover gran cantidad de nutrientes que puedan ser perjudiciales para las comunidades de macroinvertebrados.

Por último, es importante considerar que los macroinvertebrados son organismos muy importantes para otros organismos superiores como fuente de alimento, por lo que mantener una alta diversidad de estos grupos beneficiará consecuentemente a la diversidad de anfibios, peces y aves en la zona. Es importante realizar futuros estudios sobre la calidad de agua en este sistema de cultivo-riego para determinar cuáles macroinvertebrados pueden ser utilizados como indicadores de la calidad de agua en estos agroecosistemas, y pueden contribuir a brindar información para un manejo adecuado tanto del agua utilizada para regar el arroz como para su tratamiento luego del cultivo. Asimismo, es importante evaluar la composición trófica y posibles relaciones de los diferentes gremios que podrían ser condicionados por los distintos factores abióticos en el sistema.

\section{Agradecimientos.}

Los autores agradecen a la Fundación Rufford Small Grants por haber financiado este estudio. A las organizaciones Idea Wild y Neotropical Grassland Conservancy por haber donado parte del equipo de campo necesario para realizar la tesis. A la Municipalidad Distrital de Vice por haber colaborado con fondos para la realización de algunos de los análisis de agua. A Julio Peña y a la Junta de Usuarios Sechura (Comisión de regantes) por su colaboración durante todo el proyecto. A Lady Cáceres y Stefany
Salcedo por haber colaborado en la separación de las muestras de macroinvertebrados en laboratorio. A Monika Springer por sus valiosos y generosos aportes en la identificación de los invertebrados. Al Instituto del Bien Común por haber permitido que utilizara su laboratorio durante el año 2011 para analizar parte de mis muestras. A Alfredo Delgado, por ser mi compañero de campo y acompañarme en los muestreos. A Federico Rizo Patrón por su apoyo incondicional, su ayuda en el campo y colaboración en la identificación de los macroinvertebrados.

\section{Literatura citada.}

Acosta M., Mugica L., Blanco D. E., López-Lanús B., Antunes R. D., Doodnath L. W. \& Hurtado J. 2010. Birds of Rice Fields in the Americas. Waterbirds. 33(1): 105-122.

Acosta R., Ríos B., Rieradevall M. \& Prat N. 2009. Propuesta de un protocolo de evaluación de la calidad ecológica de ríos andinos (C.E.R.A) y su aplicación a dos cuencas en Ecuador y Perú. Limnetica. 28 (1): 3564.

Amorós S. K. \& Ota S. N. 2002. Evaluación de Fauna Silvestre en los Humedales de Sechura. Informe Final. . APECO, Lima, Perú.

Azrina M. Z., Yap C. K., Rahim Ismail A., Ismail A. \& Tan S. G. 2006. Anthropogenic impacts on the distribution and biodiversity of benthic macroinvertebrates and water quality of the Langat River, Peninsular Malaysia. Ecotoxicol Environ Saf. 64(3): 337-347.

Bambaradeniya C. N. B. 2000. Ecology and biodiversity in an irrigated rice field ecosystem in Sri Lanka. University of Peradeniya, Sri Lanka. 525.

Bambaradeniya C. N. B. \& Amarasinghe F. P. 2003. Biodiversity Associated with the Rice Field Agroecosystem in Asian Countries: A Brief Review, Colombo, Sri Lanka.

Blanco D. E. 2011. Conservación de los recursos acuáticos y la biodiversidad en arroceras del noreste de Argentina. Fundación para la Conservación y el Uso Sustentable de los Humedales, Buenos Aires. 108.

Blanco D. E., López-Lanús B., Antunes Dias R., Azpiroz A. \& Rilla F. 2006. Uso de arroceras por chorlos y playeros migratorios en el sur de América del Sur. Implicancias de conservación y manejo. Wetlands International, Buenos Aires, Argentina. 114.

Blázquez M. 2005. El manejo del riego en el cultivo de arroz. OTS, San José. 18.

Castillo L. E. 2000. Pesticide impact of intensive banana production on aquatic ecosystems in Costa Rica. Stockholm University, Stockholm, Sweden. 18.

Chao A. \& Shen T. J. 2009. User's Guide for Program SPADE. Species Prediction And Diversity Estimation, Taiwan.

Comin F. A., Romero B. A., Hernandez O. \& Menendez M. 2001. Restoration of Wetlands from Abandoned Rice Fields for Nutrient Removal, and Biological and Community Landscape Diversity. Restoration Ecology. 9(2): 207-208.

Curcó A., Canicio A., Escolano M. A., Fores E., Ibánez C., Luque P. \& Riera X. 2001. Mejora de la gestión del hábitat en la Zepa del Delta del Ebro (Cataluña, España). SEO/Birdlife, Cataluña. 151. 
Dominguez E. \& Fernandez H. 2009. Macroinvertebrados Bentónicos Sudamericanos: Sistemática y Biología. Fundación Miguel Lillo, Tucumán. 656.

FAO. 2004. All about rice. http://www.fao.org/rice2004/en/aboutrice.htm FAO.

Harding J. S., Young R. G., Hayes K. A. \& Stark J. D. 1999. Changes in agricultural intensity and river health along a river continuum. Freshwater Biology. 42: 345-357.

Huamantico A. A. \& Ortiz W. 2010. Clave de géneros de larvas de Trichoptera (Insecta) de la Vertiente Occidental de los Andes, Lima, Perú. Revista Peruana Biología. 17(1): 075- 080.

Iannacone J., Mansilla J. \& Ventura K. 2003. Macroinvertebrados en las lagunas de puerto viejo, Lima - Perú. Ecología Aplicada. 2(1): 116-124.

Iannacone O. J. \& Alvarino F. L. 2002. Evaluación del riesgo ambiental del insecticida cartap en bioensayos con tres invertebrados. Agric. Téc. 62(3): 366-374

INRENA. 1994. Mapa ecológico del Perú: guía explicativa. Ministerio de Agricultura, INRENA, República del Perú. I. Ministerio de Agricultura. 228.

Leitão S., Pinto P., Pereira T. \& Brito M. F. 2007. Spatial and temporal variability of macroinvertebrate communities in two farmed Mediterranean rice fields. Aquatic Ecology. 41: 373-386.

Martínez N. 2002. Evaluación de la calidad ambiental de un ecosistema de Manglar empleando macroinvertebrados acuáticos como bioindicadores. Laboratorio de Entomología. Facultad de Ciencias Biológicas, UNMSM, Lima, Perú.

Moreno C. E. 2001. Métodos para medir la biodiversidad. M\&T-Manuales y Tesis SEA, Zaragoza. 84.

Paredes C., Iannacone J. \& Alvariño L. 2007. Biodiversidad de invertebrados de los humedales de Puerto Viejo, Lima, Perú. Neotropical Helminthology. 11(1): 21-30.

Prat N., Ríos B., Acosta R. \& Rieradevall M. 2009. Los macroinvertebrados como indicadores de calidad de las aguas, Págs: 654. In: E. Domínguez \& H. Fernández (eds.). Macroinvertebrados bentónicos sudamericanos. Sistemática y biología. Fundación Miguel Lillo, Tucuman, Argentina.
Rizo-Patrón F. 2003. Estudio de los arrozales del Proyecto Tamarindo: agroquímicos y macroinvertebrados bentónicos en relación al Parque Nacional Palo Verde, Guanacaste, Costa Rica. Universidad Nacional, Heredia. 91.

Rizo-Patrón F., Kumar A., McCoy M. B. C., Springer M. \& Trama F. A. 2013. Macroinvertebrate communities as bioindicators of water quality in conventional and organic irrigated rice fields in Guanacaste, Costa Rica. Ecological Indicators. 29 68-78.

Rizo-Patrón F. \& Trama F. A. 2008. Contaminantes agrícolas y sus efectos sobre la vida silvestre en arrozales de Costa Rica. Wetlands International, Buenos Aires, Argentina. 1-10.

Roldán G. 1999. Los macroinvertebrados y su valor como indicadores de la calidad de agua. Revista Académica Colombiana Ciencias. 23(88): 376-387.

Roldán G. A. 2003. Bioindicación de la calidad del agua en Colombia. Uso del método BMWP/Col. Editorial Universidad de Antioquia, Medellin, Colombia. 170.

Statpoint Technologies I. 2007. Statgraphics Centurion. Statpoint Technologies, Inc., USA.

Tabilo-Valdivieso E., McCoy M. \& Fallas J. 1999. Comparación de técnicas para el inventario de humedales en Costa Rica. Vida Silvestre Neotropical. 7(1): 35-38.

Trama F. A., Mejía A. M. \& Rizo-Patrón F. en prep. Macroinvertebrados indicadores de la calidad de agua en arrozales de la cuenca baja del río Piura, Perú.

Trama F. A., Rizo-Patrón F. \& McCoy M. B. C. 2008. El cultivo de arroz bajo riego y las aves playeras migratorias en Perú y Costa Rica, Págs: 13. In: V. M. de la Balze \& D. E. Blanco (eds.). Primer taller para la Conservación de Aves Playeras Migratorias en Arroceras del Cono Sur. Wetlands International, Buenos Aires, Argentina.

Trama F. A., Rizo-Patrón F. \& Springer M. 2009. Macroinvertebrados bentónicos del humedal de Palo Verde, Costa Rica. Revista Biología Tropical. 57(1): 275-284.

\section{ANEXOS: Tablas y figuras citadas en el texto}

Tabla 1.- Localización de los puntos de muestreo de macroinvertebrados.

\begin{tabular}{|c|c|c|c|c|}
\hline Punto de muestreo & No. punto & Nombre del punto & Latitud & Longitud \\
\hline Canal de riego & P1 & $\mathrm{P} 1$ & S 5 25’ 20.4” & W 80 46’08.1" \\
\hline Canal de drenaje principal & $\mathrm{P} 2$ & $\mathrm{P} 2$ & S 5 $31^{\prime} 31.0^{\prime \prime}$ & W $80^{\circ} 48^{\prime} 26.0^{\prime \prime}$ \\
\hline Canal Sechura & P3 & P3 & S 5o $32^{\prime} 52.4^{\prime \prime}$ & W $80^{\circ} 49^{\prime} 42.8^{\prime \prime}$ \\
\hline Parcela VAC & VAC & $\begin{array}{l}\text { Entrada } \\
\text { Salida }\end{array}$ & $\begin{array}{l}\text { S 5 } 30 ’ 31.0^{\circ} \\
\text { S 5o } 35^{\prime} 33.0^{\prime \prime}\end{array}$ & $\begin{array}{l}\text { W } 80^{\circ} 47^{\prime} 58.6^{\prime \prime} \\
\text { W } 80^{\circ} 47^{\prime} 57.3^{\prime \prime}\end{array}$ \\
\hline Parcela MVI & MVI & $\begin{array}{l}\text { Entrada } \\
\text { Salida }\end{array}$ & $\begin{array}{l}\text { S 5 } 5^{\circ} 28^{\prime} 41.0^{\prime \prime} \\
\text { S 5 } 5^{\circ} 28,42.9^{\prime \prime}\end{array}$ & $\begin{array}{l}\text { W } 80^{\circ} 47^{\prime} 17.4 " \\
\text { W } 80^{\circ} 47^{\prime} \\
16.1 "\end{array}$ \\
\hline Parcela JTT & JTT & $\begin{array}{l}\text { Entrada } \\
\text { Salida }\end{array}$ & $\begin{array}{l}\text { S 5 } 5^{\circ} 26^{\prime} 10.66^{\prime \prime} \\
\text { S 5o } 26^{\prime} 11.2^{\prime \prime}\end{array}$ & $\begin{array}{l}\text { W } 80^{\circ} 46,31.9 " \\
\text { W } 80^{\circ} 46,33.0 "\end{array}$ \\
\hline
\end{tabular}


Tabla 2. Composición de los macroinvertebrados acuáticos registrados durante el estudio, indicando la abundancia relativa por mes para cada punto de muestreo principal y cada parcela individual (con las correspondientes entradas y salidas) dentro del sistema de riego.

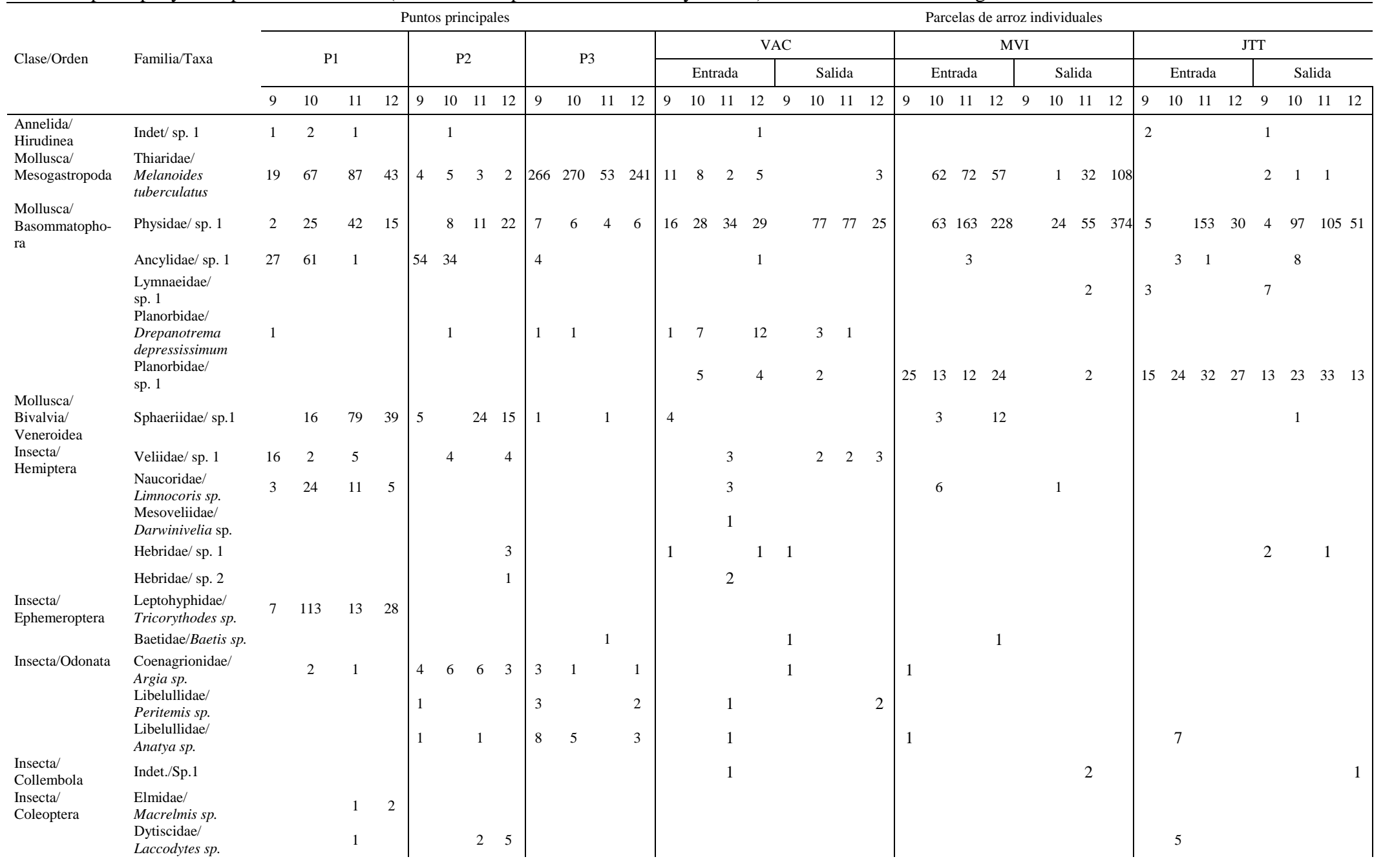


Tabla 2. Composición de los macroinvertebrados acuáticos registrados durante el estudio, indicando la abundancia relativa por mes para cada punto de muestreo principal y cada parcela individual (con las correspondientes entradas y salidas) dentro del sistema de riego.

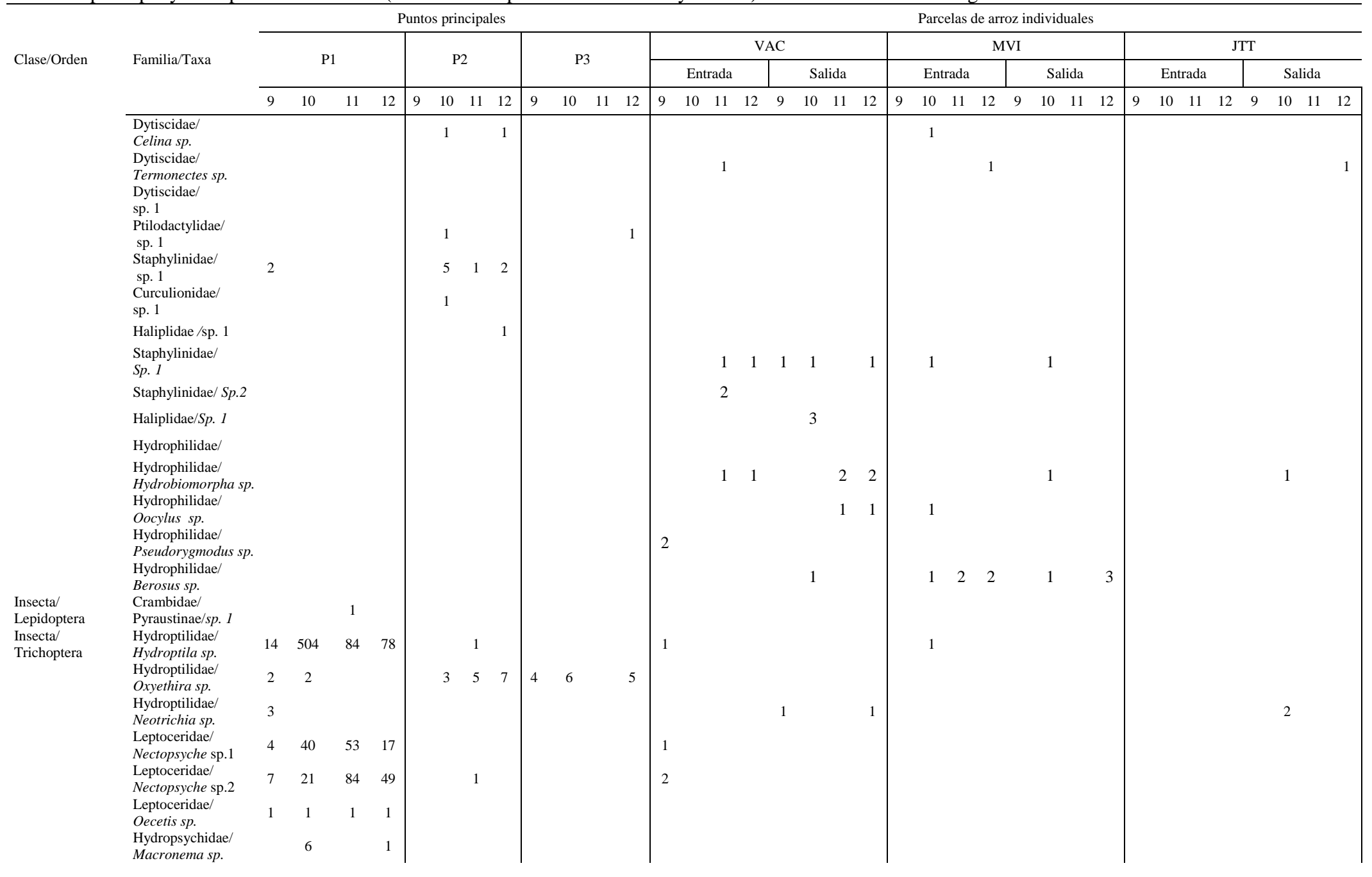


Tabla 2. Composición de los macroinvertebrados acuáticos registrados durante el estudio, indicando la abundancia relativa por mes para cada punto de muestreo principal y cada parcela individual (con las correspondientes entradas y salidas) dentro del sistema de riego.

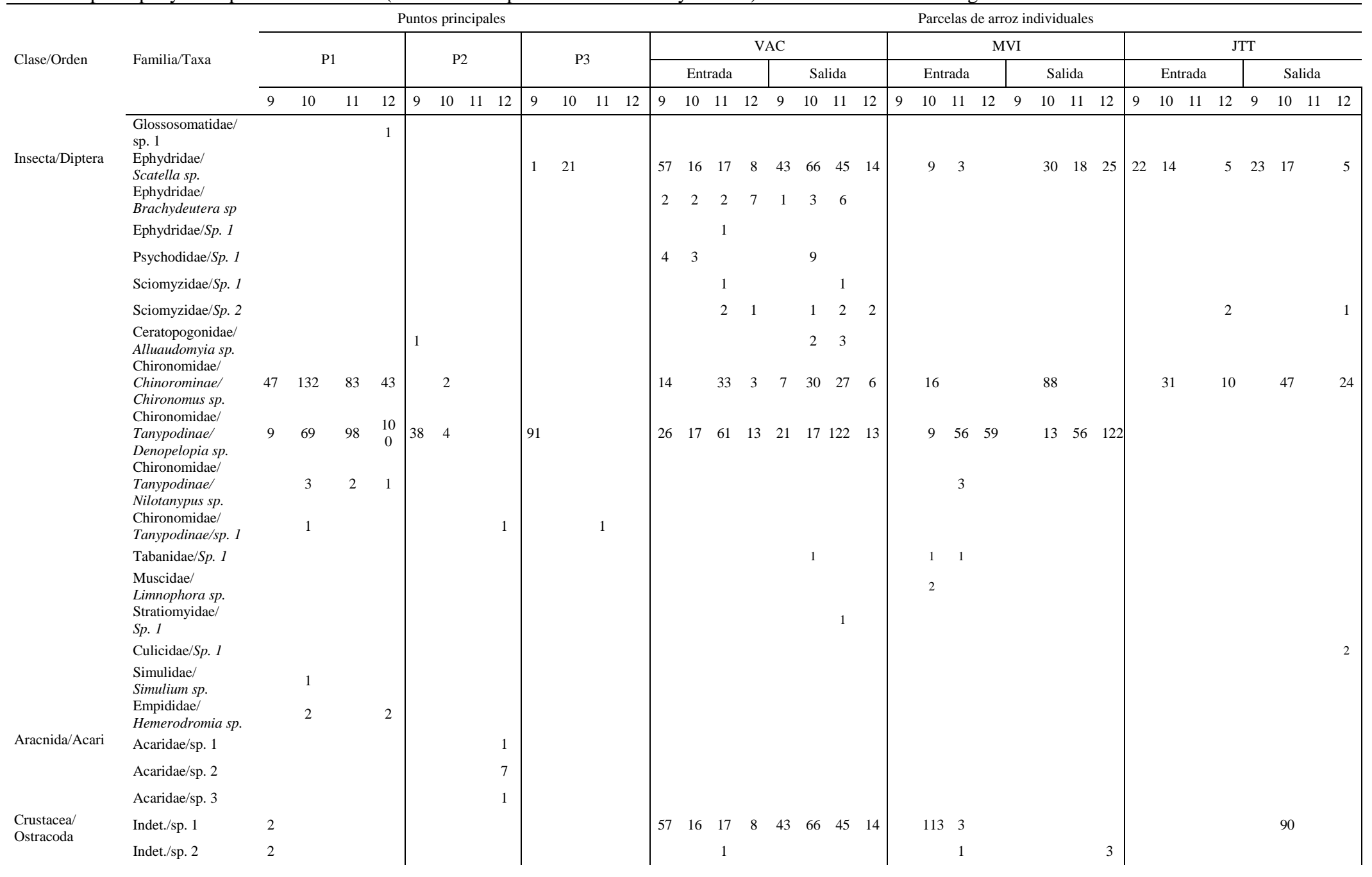


Tabla 2. Composición de los macroinvertebrados acuáticos registrados durante el estudio, indicando la abundancia relativa por mes para cada punto de muestreo principal y cada parcela individual (con las correspondientes entradas y salidas) dentro del sistema de riego.

\begin{tabular}{|c|c|c|c|c|c|c|c|c|c|c|c|c|c|c|c|c|c|c|c|c|c|c|c|c|c|c|}
\hline \multirow{4}{*}{ Clase/Orden } & \multirow{4}{*}{ Familia/Taxa } & \multicolumn{10}{|c|}{ Puntos principales } & \multicolumn{15}{|c|}{ Parcelas de arroz individuales } \\
\hline & & \multirow{2}{*}{\multicolumn{4}{|c|}{ P1 }} & \multirow{2}{*}{\multicolumn{3}{|c|}{ P2 }} & \multirow{2}{*}{\multicolumn{3}{|c|}{ Р3 }} & \multicolumn{5}{|c|}{ VAC } & \multicolumn{5}{|c|}{ MVI } & \multicolumn{5}{|c|}{ JTT } \\
\hline & & & & & & & & & & & & \multicolumn{3}{|c|}{ Entrada } & \multicolumn{2}{|r|}{ Salida } & \multicolumn{3}{|c|}{ Entrada } & \multicolumn{2}{|r|}{ Salida } & \multicolumn{3}{|c|}{ Entrada } & \multicolumn{2}{|l|}{ Salida } \\
\hline & & 9 & 10 & 11 & 12 & 9 & $10 \quad 11$ & 112 & 9 & 1011 & 12 & 9 & $10 \quad 11$ & 12 & 9 & $10 \quad 11 \quad 12$ & 9 & 1011 & 12 & $9 \quad 10$ & $10 \quad 11 \quad 12$ & 9 & $\begin{array}{lll}10 & 11 & 12\end{array}$ & 12 & $9 \quad 10 \quad 11$ & 12 \\
\hline \multirow{2}{*}{ Conchostraca } & Indet./sp. 1 & 1 & & & & & & & & & & & & & & & & & & & & \multirow{7}{*}{\multicolumn{5}{|c|}{1}} \\
\hline & Indet./sp. 2 & & & & & & & & & & & & & & & & & & & & & & & & & \\
\hline \multirow[t]{3}{*}{ Decapoda } & Indet./sp. 1 & & 2 & & & & 3 & 3 & & & 12 & & & & & & & & & & & & & & & \\
\hline & Indet./sp. 2 & & & & & & 11 & 2 & & & & & & & & & & & & & & & & & & \\
\hline & Daphnia sp. & & & & & & & & & & & & 1 & & 46 & 46 & & & & & & & & & & \\
\hline Indeterminado & & & 1 & & & 4 & 4 & 1 & & 1 & 1 & & & & & & & & & & & & & & & \\
\hline Indeterminado & & & & & 1 & & & & & & & & & & & & & & & & & & & & & \\
\hline \multicolumn{2}{|c|}{ Riqueza media de Familias } & 13 & 15 & 14 & 11 & 6 & 118 & 11 & 8 & 4 & 6 & 8 & 9 & 10 & 3 & 8 & 0 & 8 & 5 & 0 & 3 & 3 & 3 & 2 & 8 & 4 \\
\hline \multirow{2}{*}{\multicolumn{2}{|c|}{$\begin{array}{l}\text { Riqueza total de Familias por punto de } \\
\text { muestreo } \\
\text { Riqueza media de taxa }\end{array}$}} & \multicolumn{4}{|c|}{23} & \multicolumn{3}{|c|}{19} & \multicolumn{3}{|c|}{11} & \multicolumn{3}{|c|}{23} & \multicolumn{2}{|r|}{18} & & 13 & & & 7 & & 5 & & 13 & \\
\hline & & 19 & 22 & 19 & 27 & $8 \quad 16$ & $16 \quad 12$ & 218 & 11 & 75 & 8 & 14 & 1121 & 15 & 6 & $15 \quad 15 \quad 12$ & 1 & $14 \quad 11$ & 8 & 0 & 86 & 4 & 5 & 5 & $\begin{array}{lll}5 & 10 & 3\end{array}$ & 8 \\
\hline $\begin{array}{l}\text { Riqueza total de } \\
\text { muestreo }\end{array}$ & xa por punto de & & 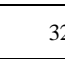 & & & & 31 & & & 15 & & & 37 & & & 26 & & 20 & & & 11 & & 11 & & 8 & \\
\hline Riqueza total de & xa total por parcela & & & & & & & & & & & & & 4 & 14 & & & & 22 & & & & & 18 & & \\
\hline Abundancia me & & 267 & 1959 & 1027 & 652 & 17815 & 152111 & 11140 & 674 & $608 \quad 118$ & 534 & 143 & $89 \quad 170$ & 87 & 118 & 21734072 & 25 & 300318 & 383 & $\begin{array}{ll}0 & 1\end{array}$ & 158166681 & 44 & $79 \quad 1867$ & 74 & $43 \quad 288139$ & $\begin{array}{l}9 \\
100\end{array}$ \\
\hline Abundancia tot & por punto de muestreo & & 23 & & & & 332 & & & 1029 & & & 489 & & & 746 & & 1027 & & & 1006 & & 383 & & 570 & \\
\hline Abundancia tot & oor parcela & & & & & & & & & & & & & 12 & 235 & & & & 2032 & & & & & 953 & & \\
\hline
\end{tabular}


Agosoto - Diciembre 2013

Tabla 3. Riqueza y abundancia de macroinvertebrados en \% para cada orden colectado en cada punto de muestreo. Datos presentados en porcentaje.

\begin{tabular}{|c|c|c|c|c|c|c|c|c|c|c|c|c|}
\hline \multirow{2}{*}{ Clase /Orden } & \multicolumn{6}{|c|}{ Riqueza de macroinvertebrados } & \multicolumn{6}{|c|}{ Abundancia de Macroinvertebrados } \\
\hline & P1 & P2 & P3 & VAC & MVI & JTT & P1 & P2 & P3 & VAC & MVI & JTT \\
\hline Hirudinea & 2,94 & 3,23 & & 1,61 & 2,94 & & 0,21 & 0,63 & & 0,08 & 0,15 & \\
\hline Basommatophora & 8,82 & 9,68 & 18,75 & 11,29 & 14,71 & 26,09 & 7,47 & 41,04 & 13,92 & 25,95 & 44,60 & 62,62 \\
\hline Mesogastropoda & 2,94 & 3,23 & 6,25 & 3,23 & 5,88 & 4,35 & 9,22 & & & 2,29 & 16,18 & 0,38 \\
\hline Veneroidea & 2,94 & 3,23 & 6,25 & 1,61 & 2,94 & 4,35 & 5,72 & 13,87 & 1,00 & 0,28 & 1,01 & 0,19 \\
\hline Ephemeroptera & 2,94 & & 6,25 & 3,23 & 2,94 & & 6,86 & & 0,50 & 0,16 & 0,07 & \\
\hline Trichoptera & 23,53 & 9,68 & 6,25 & 4,84 & 2,94 & 4,35 & 41,80 & 5,36 & 7,50 & 0,32 & 0,07 & 0,38 \\
\hline Odonata & 2,94 & 9,68 & 18,75 & 9,68 & & 4,35 & 0,13 & 7,04 & 12,83 & 0,57 & & \\
\hline Coleoptera & 11,76 & 19,35 & 6,25 & 20,97 & 20,59 & 13,04 & 0,29 & 6,04 & 0,50 & 2,09 & 1,09 & 0,57 \\
\hline Collembola & & & & 1,61 & 2,94 & 4,35 & & & & 0,08 & 0,13 & 0,19 \\
\hline Hemiptera & 5,88 & 9,68 & & 9,68 & 5,88 & 4,35 & 2,79 & 3,47 & & 1,55 & 0,53 & 0,47 \\
\hline Lepidoptera & 2,94 & & & & & & 0,04 & & & & & \\
\hline Diptera & 17,65 & 12,90 & 18,75 & 29,03 & 29,41 & 30,43 & 25,36 & 14,35 & 56,75 & 59,15 & 28,31 & 18,15 \\
\hline Ostracoda & 5,88 & & & & 8,82 & 4,35 & 0,15 & & & & 7,87 & 17,01 \\
\hline Acari/acarina & & 9,68 & & & & & & 2,84 & & & & \\
\hline Decapoda & 5,88 & 9,68 & 12,50 & 3,23 & & & 0,13 & 5,99 & 7,00 & 7,45 & & \\
\hline Conchostraca & 2,94 & & & & & & 0,04 & & & & & \\
\hline
\end{tabular}

Tabla 4. Pasos para el cultivo de arroz en la campaña chica (agosto-diciembre 2010) para las tres parcelas evaluadas.

\begin{tabular}{|c|c|c|c|}
\hline $\begin{array}{l}\text { Pasos en el cultivo de } \\
\text { arroz }\end{array}$ & VAC & MVI & JTT \\
\hline Preparación del suelo & $\begin{array}{l}\text { Preparación de semillero } \\
\text { Gradeo y luego nivelado del } \\
\text { terreno }\end{array}$ & $\begin{array}{l}\text { Preparación de semillero } \\
\text { Gradeo con niveladora } \\
\text { Nivelación del suelo e inundación } \\
\text { Fangueo }\end{array}$ & $\begin{array}{l}\text { Preparación de la tierra y } \\
\text { semillero } \\
\text { Fangueo }\end{array}$ \\
\hline & Trasplante con agua & Trasplante con agua & Trasplante con agua \\
\hline Inundación y trasplante & $\begin{array}{l}\text { Parcelas con una entrada de } \\
\text { agua y una salida }\end{array}$ & $\begin{array}{l}\text { Parcelas con una entrada de agua y } \\
\text { una salida }\end{array}$ & $\begin{array}{l}\text { Parcelas con una entrada de agua } \\
\text { y una salida }\end{array}$ \\
\hline Aplicación inicial & $\begin{array}{l}\text { Aplicación de herbicida ( Satur } \\
\text { y Chanteley) }\end{array}$ & $\begin{array}{l}\text { Aplicación de herbicida a los } 8 \text { días } \\
\text { de la siembra (Machete) } 3 \text { bolsas por } \\
\text { ha. }\end{array}$ & $\begin{array}{l}\text { No usa herbicidas } \\
\text { Saca hierbas a mano }\end{array}$ \\
\hline Abonada inicial & $\begin{array}{l}2 \text { sacos de urea, } 2 \text { de sulfato y } \\
P, K(35,15)\end{array}$ & $\begin{array}{l}\text { Luego de } 5 \text { días se seca la parcela } \\
\text { para abonar } \\
8 \text { sacos de urea por ha }\end{array}$ & $\begin{array}{l}3 \text { sacos urea y } 1 \text { de sulfato por ha } \\
\text { Uso de abonos foliares } \\
\text { (Promalina) }\end{array}$ \\
\hline Segunda abonada & & 8 sacos de urea y 2 de sulfato por ha & 2 sacos de urea y 2 de sulfato \\
\hline Manejo de Plagas & $\begin{array}{l}\text { Dorsan (insecticida) } 60 \\
\text { mg/mochila y Balazo } \\
\text { (herbicida) } 35 \text { mg/mochila }\end{array}$ & $\begin{array}{l}\text { Aplicación de insecticida } \\
\text { Aplicación de fungicida }\end{array}$ & $\begin{array}{l}\text { Aplicación de insecticida } \\
\text { (cipermetrina) } \\
\text { Aplicación de hirundinae }\end{array}$ \\
\hline Tercera abonada & & & 3 sacos de urea y 1 de sulfato \\
\hline Cosecha & Cosecha a mano & Cosecha a mano & Cosecha a mano \\
\hline
\end{tabular}


Tabla 5. Valores medios de los parámetros fisicoquímicos para cada punto principal y parcelas de arroz individuales, en cada mes de muestreo y para todo el estudio por punto de muestreo. Se detallan los valores por mes y los valores medios (desviación estándar) para cada punto y parámetro.

\begin{tabular}{|c|c|c|c|c|c|c|c|}
\hline $\begin{array}{l}\text { Punto de } \\
\text { muestreo }\end{array}$ & $\begin{array}{l}\text { Profundidad } \\
\quad(\mathrm{cm})\end{array}$ & $\begin{array}{l}\text { Conductividad } \\
\text { eléctrica }(\mu S)\end{array}$ & $\begin{array}{l}\text { Salinidad } \\
\text { (ppm) }\end{array}$ & $\mathrm{pH}$ & $\begin{array}{c}\text { Temperatura } \\
\left({ }^{\circ} \mathrm{C}\right)\end{array}$ & $\begin{array}{c}\text { Oxígeno } \\
\text { Disuelto } \\
\text { (mg/l) }\end{array}$ & $\begin{array}{l}\text { NTU } \\
\text { turbidez }\end{array}$ \\
\hline \multicolumn{8}{|l|}{ Agosto } \\
\hline P1 & 65.0 & 357.2 & 0.3 & 8.15 & 22.5 & 3.92 & 1.53 \\
\hline P2 & 34.0 & 12890.0 & 7.4 & 7.61 & 26.0 & 2.53 & 1.70 \\
\hline P3 & 100.0 & - & - & 7.77 & 27.8 & 3.06 & 1.60 \\
\hline \multicolumn{8}{|c|}{ Septiembre } \\
\hline $\mathrm{P} 1$ & 76.0 & 777.0 & 0.4 & 8.19 & 20.7 & 5.52 & 1.21 \\
\hline P2 & 40.0 & 5300.0 & 2.9 & 7.67 & 20.7 & 2.92 & 1.22 \\
\hline P3 & 50.0 & 4991.0 & 2.7 & 7.87 & 22.4 & 6.16 & 1.14 \\
\hline Ent VAC & 15.0 & 1315.00 & 0.7 & 8.74 & 21.4 & 10.52 & 1.38 \\
\hline Sal VAC & 12.0 & 1335.00 & 0.7 & 7.79 & 23.2 & 3.75 & 1.79 \\
\hline Ent MVI & 10.0 & 519.00 & 0.3 & 8.14 & 22.3 & 7.61 & 1.29 \\
\hline Sal MVI & 12.0 & 792.00 & 0.30 & 7.80 & 24.00 & 4.40 & 1.18 \\
\hline Ent JTT & 22.0 & 690.00 & 0.40 & 9.00 & 27.90 & 8.20 & 1.51 \\
\hline Sal JTT & 24.0 & 795.00 & 0.40 & 9.12 & 29.00 & 6.30 & 1.40 \\
\hline \multicolumn{8}{|l|}{ Octubre } \\
\hline $\mathrm{P} 1$ & 60.0 & 791.0 & 0.4 & 8.69 & 22.5 & 7.63 & 1.21 \\
\hline P2 & 64.0 & 5140.0 & 2.8 & 8.18 & 22.3 & 4.29 & 1.29 \\
\hline P3 & 80.0 & 5490.0 & 3.0 & 8.48 & 22.9 & 4.93 & 1.18 \\
\hline Ent VAC & 10.0 & 1052.00 & 0.5 & 8.14 & 26.9 & 5.69 & 1.29 \\
\hline Sal VAC & 14.0 & 1150.00 & 0.6 & 8.7 & 28.7 & 5.46 & 1.61 \\
\hline Ent MVI & 8.0 & 150.70 & 0.2 & 9.81 & 26.7 & 7.28 & 1.58 \\
\hline Sal MVI & 12.0 & 2342.00 & 1.20 & 9.16 & 32.20 & 8.68 & 1.45 \\
\hline Ent JTT & 12.0 & 779.00 & 0.40 & 9.11 & 30.90 & 10.27 & 1.45 \\
\hline Sal JTT & 15.0 & 585.00 & 0.30 & 9.20 & 30.00 & 8.79 & 1.67 \\
\hline \multicolumn{8}{|c|}{ Noviembre } \\
\hline $\mathrm{P} 1$ & 64.0 & 95.0 & 0.1 & 8.55 & 22.5 & 4.50 & 1.18 \\
\hline P2 & 70.0 & 4450.0 & 3.1 & 8.14 & 21.8 & 4.45 & 2.37 \\
\hline P3 & 85.0 & 6140.0 & 3.3 & 8.05 & 22.0 & 2.95 & 1.46 \\
\hline Ent VAC & 5.0 & 839.00 & 0.4 & 7.6 & 28.3 & 1.85 & 2.37 \\
\hline Sal VAC & 3.0 & 440.00 & 0.2 & 7.98 & 27.8 & 6.25 & 2.37 \\
\hline Ent MVI & 6.0 & 722.00 & 0.4 & 9.09 & 25.9 & 5.13 & 1.23 \\
\hline Sal MVI & 15.0 & 2469.00 & 1.30 & 8.03 & 28.00 & 6.65 & 1.18 \\
\hline Ent JTT & 16.0 & 705.00 & 0.30 & 7.77 & 20.40 & 3.45 & 1.55 \\
\hline Sal JTT & 12.0 & 835.00 & 0.40 & 7.81 & 20.90 & 2.82 & 1.28 \\
\hline \multicolumn{8}{|l|}{ Diciembre } \\
\hline P1 & 59.0 & 733.0 & 0.4 & 8.96 & 27.4 & 10.15 & 1.35 \\
\hline P2 & 79.0 & 5750.0 & 3.1 & 7.97 & 23.4 & 3.31 & 1.42 \\
\hline P3 & 80.0 & 6980.0 & 3.8 & 7.67 & 23.3 & 3.55 & 1.75 \\
\hline Ent VAC & 5.0 & 845.00 & 0.4 & 9.18 & 19.7 & 1.83 & 2.00 \\
\hline Sal VAC & 3.0 & 979.00 & 0.5 & 8.01 & 20.2 & 2.07 & 1.75 \\
\hline Ent MVI & 6.0 & 875.00 & 0.4 & 7.84 & 20.1 & 1.06 & 2.23 \\
\hline Sal MVI & 5.0 & 2505.00 & 1.30 & 8.13 & 20.30 & 2.70 & 1.28 \\
\hline Ent JTT & 12.0 & 574.00 & 0.40 & 7.71 & 22.70 & 3.82 & 2.08 \\
\hline Sal JTT & 20.0 & 881.00 & 0.30 & 7.77 & 22.30 & 3.96 & 1.91 \\
\hline \multicolumn{8}{|c|}{ Promedio (desviación estándar) por punto y parcela para todo el estudio } \\
\hline P1 & $64.8(6.760)$ & $550.64(311.16) \mathrm{a}$ & $0.32(0.13) \mathrm{a}$ & $8.5(0.34) \mathrm{a}$ & $23.12(2.52)$ & $6.34(2.55) \mathrm{a}$ & $1.29(0.15)$ \\
\hline $\mathrm{P} 2$ & $57.4(19.49)$ & $6706.00(3488.40) b$ & $3.86(1.98) \mathrm{b}$ & $7.9(0.26) \mathrm{b}$ & $22.84(2.02)$ & $3.50(0.84) \mathrm{b}$ & $1.60(0.47)$ \\
\hline P3 & $79.0(16.02)$ & $5900.25(859.90) \mathrm{b}$ & $3.20(0.47) \mathrm{b}$ & $8.0(0.35) \mathrm{b}$ & $23.68(0.57)$ & $4.13(1.44)$ & $1.43(1.29)$ \\
\hline Ent VAC & $8.8(4.8)$ & $1012.8(224.5)$ & $0.5(0.1)$ & $8.4(0.7)$ & $24.1(4.2)$ & $5.0(4.1)$ & $1.8(0.5)$ \\
\hline Sal VAC & $8.0(5.8)$ & $976.0(385.8)$ & $0.5(0.2)$ & $8.1(0.4)$ & $25.0(4.0)$ & $4.4(1.9)$ & $1.9(0.3)$ \\
\hline Ent MVI & $7.5(1.9)$ & 566.7 (313.3)a & $0.3(0.1) \mathrm{a}$ & $8.7(0.9)$ & $23.8(3.1)$ & $5.3(3.0)$ & $1.6(0.5)$ \\
\hline Sal MVI & $11.0(4.2)$ & 2027.0 (826.3)b & $1.0(0.5) \mathrm{b}$ & $8.3(0.6)$ & $26.1(5.1)$ & $5.6(2.6)$ & $1.3(0.1)$ \\
\hline
\end{tabular}


Agosoto - Diciembre 2013

Tabla 5. Valores medios de los parámetros fisicoquímicos para cada punto principal y parcelas de arroz individuales, en cada mes de muestreo y para todo el estudio por punto de muestreo. Se detallan los valores por mes y los valores medios (desviación estándar) para cada punto y parámetro.

\begin{tabular}{lccccccc}
\hline $\begin{array}{l}\text { Punto de } \\
\text { muestreo }\end{array}$ & $\begin{array}{c}\text { Profundidad } \\
(\mathrm{cm})\end{array}$ & $\begin{array}{c}\text { Conductividad } \\
\text { eléctrica }(\mu \mathrm{S})\end{array}$ & $\begin{array}{c}\text { Salinidad } \\
(\mathrm{ppm})\end{array}$ & $\mathrm{pH}$ & $\begin{array}{c}\text { Temperatura } \\
\left({ }^{\circ} \mathrm{C}\right)\end{array}$ & $\begin{array}{c}\text { Oxígeno } \\
\text { Disuelto } \\
(\mathrm{mg} / \mathrm{l})\end{array}$ & $\begin{array}{c}\text { NTU } \\
\text { turbidez }\end{array}$ \\
\hline Ent JTT & $15.5(4.7)$ & $687.0(84.8)$ & $0.4(0.1)$ & $8.4(0.8)$ & $25.5(4.8)$ & $6.4(3.3)$ & $1.6(0.3)$ \\
Sal JTT & $17.8(5.3)$ & $774.0(130.8)$ & $0.4(0.1)$ & $8.5(0.8)$ & $25.6(4.6)$ & $5.5(2.6)$ & $1.6(0.3)$ \\
\hline VAC & $8.4(5.0) \mathrm{c}$ & $994.4(292.9) \mathrm{ab}$ & $0.5(0.2) \mathrm{ab}$ & $8.3(0.5)$ & $24.5(3.8)$ & $4.7(3.0)$ & $1.8(0.4) \mathrm{b}$ \\
MVI & $9.3(3.6) \mathrm{bc}$ & $1296.8(971.6) \mathrm{a}$ & $0.7(0.5) \mathrm{b}$ & $8.5(0.7)$ & $24.9(4.1)$ & $5.4(2.6)$ & $1.4(0.4) \mathrm{ab}$ \\
JTT & $16.6(4.8) \mathrm{a}$ & $730.5(112.1) \mathrm{b}$ & $0.4(0.1) \mathrm{a}$ & $8.4(0.7)$ & $25.5(4.4)$ & $6.0(2.8)$ & $1.6(0.3) \mathrm{a}$ \\
\hline
\end{tabular}

Nota: Sal = salida, Ent $=$ entrada. Letras diferentes en los valores medios indican diferencias estadísticas $(\mathrm{p}<0.05)$.

Tabla 6. Concentraciones de nutrientes evaluados en los puntos principales de muestreo. Se detalla el tipo de tendencia de los datos durante todo el período (+ o $-)$ y el r $r^{2}$.

\begin{tabular}{|c|c|c|c|c|c|c|c|}
\hline & Agosto & Septiembre & Octubre & Noviembre & Diciembre & $r^{2}$ & Tendencia \\
\hline \multicolumn{8}{|l|}{ Fosfatos } \\
\hline P1 & 1.14 & 0.08 & 0.12 & 0.11 & 0.1 & 0.485 & - \\
\hline P2 & 0.49 & 1.12 & 0.05 & 0.2 & 1.16 & 0.017 & + \\
\hline P3 & 0.46 & 0.6 & 0.2 & 0.12 & 0.84 & 0.023 & + \\
\hline \multicolumn{8}{|l|}{ Potasio } \\
\hline P1 & 2.53 & 2.77 & 7.11 & 6.32 & 7.02 & 0.739 & + \\
\hline P2 & 48.32 & 51.83 & 35.43 & 40.27 & 48.25 & 0.075 & - \\
\hline P3 & 30.25 & 34.12 & 23.34 & 30.67 & 35.12 & 0.046 & + \\
\hline \multicolumn{8}{|l|}{ Amonio } \\
\hline P1 & 0.12 & 0.11 & 0.1 & 0.12 & 0.1 & 0.225 & - \\
\hline P2 & 0.1 & 0.11 & 0.09 & 0.1 & 0.07 & 0.533 & - \\
\hline P3 & 0.11 & 0.16 & 0.11 & 0.14 & 0.16 & 0.254 & + \\
\hline \multicolumn{8}{|l|}{ Nitratos } \\
\hline P1 & 0.32 & 0.05 & 0.8 & 0.4 & 0.22 & 0.007 & + \\
\hline P2 & 0.4 & 0.1 & 0.1 & 1.3 & 1.62 & 0.651 & + \\
\hline P3 & 0.9 & 0.2 & 0.4 & 0.3 & 0.66 & 0.027 & - \\
\hline \multicolumn{8}{|l|}{ Nitritos } \\
\hline P1 & 0.09 & 0.07 & 0.14 & 0.16 & 0.03 & 0.485 & - \\
\hline P2 & 0.32 & 0.09 & 0.18 & 0.21 & 0.07 & 0.017 & - \\
\hline P3 & 0.11 & 0.1 & 0.16 & 0.18 & 0.09 & 0.023 & + \\
\hline \multicolumn{8}{|c|}{ Fuentes inorgánicas N (Nitratos, Nitritos y Amonio) } \\
\hline P1 & 0.53 & 0.23 & 1.04 & 0.68 & 0.35 & 0.002 & + \\
\hline $\mathrm{P} 2$ & 0.81 & 0.30 & 0.34 & 1.61 & 1.76 & 0.541 & + \\
\hline P3 & 1.11 & 0.41 & 0.67 & 0.62 & 0.91 & 0.012 & - \\
\hline
\end{tabular}




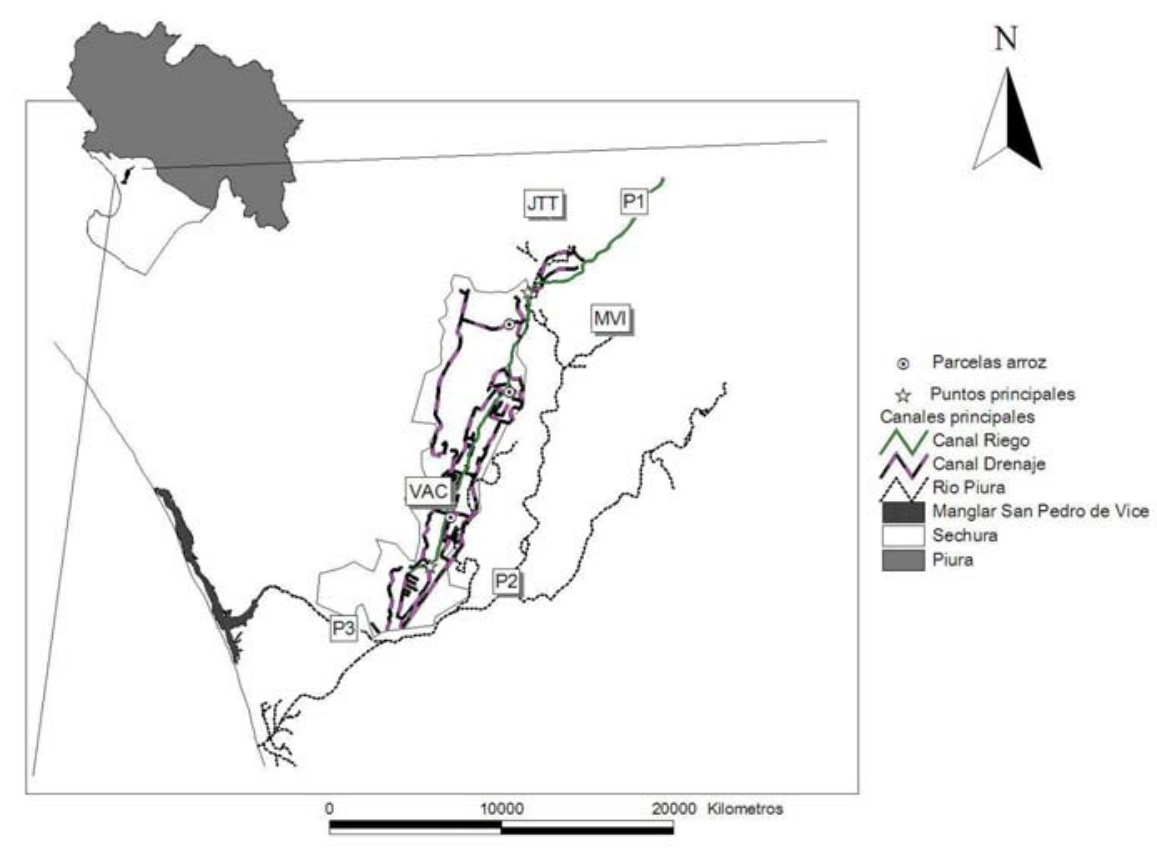

Figura 1. Comisión de Riego Muñuela margen derecha y sitio de estudio para el presente trabajo. Puntos de muestreo: P1, entrada de agua de riego; P2, Salida de agua de riego; P3, canal al manglar de San Pedro de Vice. Se detallan además las parcelas de arroz VAC, MVI y JTT. 
Agosoto - Diciembre 2013
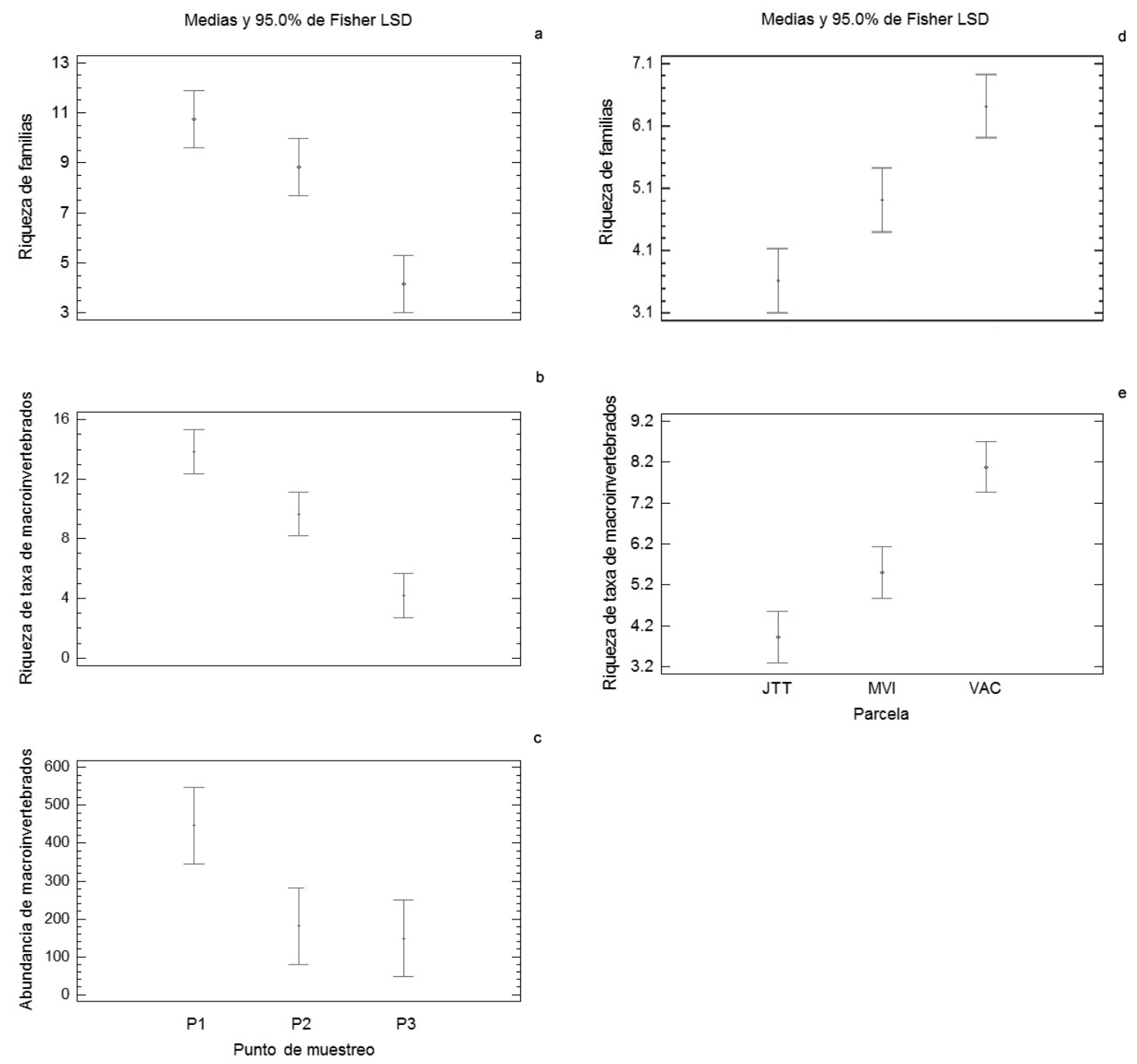

Figura 2. Análisis de varianza entre los diferentes puntos de muestreo y parcelas evaluadas para todo el estudio: a-c, variables de riqueza de familias, de taxa y abundancia de macroinvertebrados en el sistema de riego, P1, P2 y P3; d-e, Riqueza de familias y de taxa de macroinvertebrados para las tres parcelas de arroz evaluadas.

\footnotetext{
${ }^{1}$ Autor correspondiente. Centro Neotropical de Entrenamiento en Humedales - Perú. Jr. Puerto Inca \# 174 Dpto. 302. Urb. Los Olivos - Surco. Lima 33, Lima. Perú. ftrama@centroneotropical.org Universidad Nacional Agraria La Molina, Perú.

${ }^{2}$ Universidad Nacional Agraria La Molina, Perú. Email: jabel@lamolina.edu.pe
} 